\title{
Improving the Rate of Convergence of Blind Adaptive Equalization for Fast Varying Digital Communication Systems
}

\author{
Iorkyase, E.Tersoo \\ Electrical and Electronics Engineering \\ University of Agriculture, Makurdi, Nigeria
}

\author{
Michael O. Kolawole \\ Electrical and Electronics Engineering \\ Federal University of Technology, Akure \\ Akure,Nigeria
}

\begin{abstract}
The recent digital transmission systems impose the application of channel equalizers with bandwidth efficiency, which mitigates the bottleneck of intersymbol interference for high-speed data transmission-over communication channels. This leads to the exploration of blind equalization techniques that do not require the use of a training sequence. Blind equalization techniques however suffer from computational complexity and slow convergence rate. The Constant Modulus Algorithm (CMA) is a better technique for blind channel equalization. This paper examined three different error functions for fast convergence and proposed an adaptive blind equalization algorithm with variable step size based on CMA criterion. A comparison of the existing and proposed algorithms' speed of convergence shows that the proposed algorithm outperforms the other algorithms. The proposed algorithm can suitably be employed in blind equalization for rapidly changing channels as well as for high data rate applications.
\end{abstract}

Keywords- Blind Equalization; Channel Equalizer; Constant Modulus Algorithm; Intersymbol interference; Variable Step Size.

\section{INTRODUCTION}

One of the most important advantages of the digital transmission systems for voice, data and video communication is their higher reliability in noise environment in comparison with that of their analog counterparts. Both existing wired and wireless communication systems have significantly made a shift to digital transmission of data. Unfortunately, most often the digital transmission of information is accompanied with a phenomenon known as intersymbol interference (ISI). This means that the transmitted pulses are smeared out so that pulses that correspond to different symbols are not separable. ISI is a common problem in telecommunication system and wireless communication systems, such as television broadcasting, digital data communication, and cellular mobile communication systems.

In telecommunication systems, ISI occurs when the modulation bandwidth exceeds the coherent bandwidth of the radio channel where modulation pulses are spread in time. For wireless communication, ISI is caused by multipath in bandlimited time-dispersive channels, and it distorts the transmitted signal, causing bit errors at the receiver. ISI has been recognized as the major obstacle to high-speed data transmission with the required accuracy and multipath fading over radio channels.

Obviously, for a reliable digital transmission system, it is crucial to reduce the effect of ISI. This can be achieved by the technique of equalization [1, 2]. Equalization is one of the techniques that can be used to improve the received signal quality in telecommunication especially in digital communication. In a broad sense, the term equalization can be used to describe any signal processing operation that minimizes the ISI. Two of the most intensively developing areas of digital transmission, namely digital subscriber lines (DSL) and cellular communication (GSM) are strongly dependent on the realization of reliable channel equalizers [3, $4,5]$.

There are generally two approaches to equalization: conventional equalization, and "blind" equalization. In systems employing conventional equalization, a training sequence is transmitted over the channel prior to the transmission of any useful data. The training sequence is a data sequence that is a priori known to the receiver. The receiver uses the relationship between the known training sequence and the sequence it actually receives to construct an approximation of the inverse transfer function for the channel. The equalizer is then configured to use the inverse transfer function and thereby induce minimal ISI.

Conventional equalization is problematic in some communication systems, such as mobile and multi-point communication systems, because the training sequence uses up scarce bandwidth resources that could otherwise be used to transmit useful data. Such systems, therefore, often use blind equalization, which is a form of equalization that does not require the use of a training sequence.

It is desirable to improve the ability of digital communication systems to minimize ISI, including communication systems employing blind equalization. Systems achieving such reduced ISI are capable of achieving reduced data error rates at prevailing data transmission rates, or can obtain higher data transmission rates without sacrificing data integrity, in order to obtain better overall system performance [6]. 


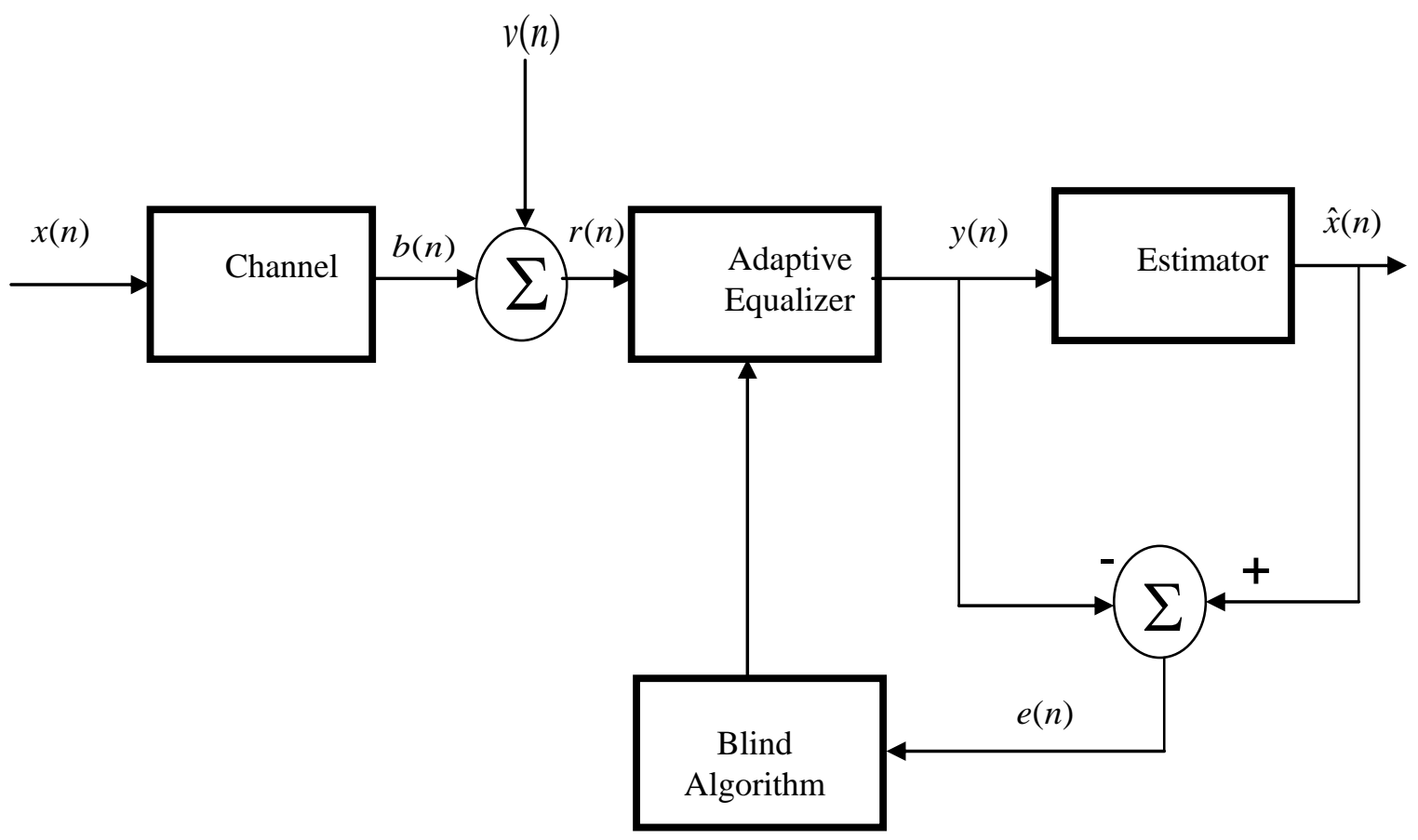

Figure 1: Baseband model of a Communication System for Channel Equalization.

In order to provide an efficiently high data rate transmission with high accuracy in digital communication without spectral wastage, advanced signal processing techniques are necessary. As earlier mentioned, several digital communication systems are inherent with rapid varying channel characteristics. The assumption that a communication channel is stationary over a transmission period is not valid. For communication systems like mobile communication this assumption results in performance degradation. There arises a need for algorithms that can exercise tracking ability in the face of fast changing characteristics of communication channels [7].

The rate of convergence becomes very pivotal in the development of any such algorithms. This paper considers an adaptive blind equalization technique based on Constant Modulus Criterion using different error functions and a comparison of their speed of convergence is made.

\section{SYSTEM MOdEL For BLIND ADAPTIVE EQUALIZATION}

The baseband Model of a communication system for channel equalization is shown in Fig 1.

In Fig. 1, our communication channel and equalizer are both modeled using a Finite Impulse Response filter (FIR). Of course, the channel and equalizer can also be modeled as Infinite Impulse Response filter (IIR).

However, it is dangerous to update the poles of an IIR filter in real-time, because it is possible that they could move outside the unit circle, hence causing instability in the system.

From Figure 1, the relationship between the input and output of the FIR channel filter is,

$$
b(n)=\sum_{k} h(k) x(n-k)
$$

The input to the equalizer is,

$$
r(n)=b(n)+v(n)
$$

This implies that,

$$
r(n)=\sum_{k} h(k) x(n-k)+v(n)
$$

where $h(k)$ is the channel response.

$x(n-k)$ is the input to the channel at time $n-k$.

$v(n)$ represents the additive noise (zero mean)

$r(n)$ is the input to the equalizer.

The output of the system $y(n)$ is given as,

$$
y(n)=w^{T} r(n)
$$

where $y(n)$ is the equalizer output and $w^{T}$ is the tap weight vector.

The equalizer model (4) forms the basis for the discussion of the blind adaptive algorithms in the following subsection.

\section{A. Constant Modulus Algorithm Criterion}

The CMA criterion may be expressed by the non-negative cost function $J_{C M A p, q}$ parameterized by positive integer, $p$ and $q$. 


$$
J_{C M A_{p, q}}=\frac{1}{p q} E\left\{|y(n)|^{p}-\left.R\right|^{q}\right\}
$$

where $R$ is a fixed constant, chosen for each form of modulation schemes, represents the statistics of the transmitted signal. $J_{C M A}$ in (5) is a gradient based algorithm [8] and works on the premise that the existing interference causes fluctuation in the amplitude of the output that otherwise has a constant modulus. For the simplest case we put $p=2$ and $q=2$, we have

$$
J_{C M A}=\frac{1}{4}\left(|y(n)|^{2}-R\right)^{2}
$$

It updates equalizer coefficients by minimizing the cost function. The steepest gradient descent algorithm [9] is obtained by taking the instantaneous gradient of $J_{C M A}$ which results in an equation that updates the system.

$$
\begin{aligned}
& w(n+1)=w(n)-\mu g(f(n)) \\
& g(f(n))=r^{*}(n) \psi(y(n)) \\
& \psi(y(n))=-\nabla_{y(n)} \frac{1}{4}\left(|y(n)|^{2}-R\right)^{2} \\
& \psi_{1}(y(n))=y(n)\left(R-|y(n)|^{2}\right)
\end{aligned}
$$

where $w(n)$ is the equalizer coefficient, $r(n)$ is the receiver input, $\mu$ is the step size constant and $\psi_{1}(y(n))$ is the error function for $C M A$

With the above expression, $\psi(y(n))$ for the error function, the usual $C M A$ becomes:

$$
\begin{aligned}
& w(n+1)=w(n)+r^{*}(n) \mu y(n)\left(R-|y(n)|^{2}\right) \\
& w_{i}(n+1)=w_{i}(n)+r^{*}(n) \mu y(n)\left(R-|y(n)|^{2}\right)
\end{aligned}
$$

where $w_{i}$ is the $i^{t h}$ tap of the equalizer. The signed error version of CMA (SE-CMA) takes

$$
\psi_{2}(y(n))=\operatorname{sgn}\left[\psi_{1}(y(n))\right]
$$

and updates the equalizer as;

$$
w(n+1)=w(n)+r^{*}(n) \mu \operatorname{sgn}\left\{y(n)\left(R-|y(n)|^{2}\right)\right\}
$$

\section{B. Proposed Error Functions}

In this section we construct three error functions $\psi_{i}(y(n)), i=3,4,5$. Assume the source to be a real BPSK (Binary Phase Shift Keying) with equiprobable alphabet and unity dispersion $R=1$. Note that the dispersion constant $R$ is chosen for each form of modulation scheme. Let the product of the squared deviations of the output be $P[y(n)]$. For real BPSK source, $P[y(n)]$ is taken as [10]:

$$
[(y(n)+1)(y(n)-1)]^{2}
$$

case of $\psi_{3}(y(n))$ and

in the

$$
[y(n)(y(n)+1)(y(n)-1)]^{2}
$$

In the case of $\psi_{4}(y(n))$

Using a step size parameter $\mu$, the updated equation can be written as

$$
\begin{aligned}
& w(n+1)=w(n)-\frac{1}{2} \mu \nabla P(y(n)) \\
& =w(n)-\frac{1}{2} \mu\left[\frac{2 \partial P(y(n))}{\partial w}\right]_{w=w(n)} \\
& =w(n)-\mu \frac{\partial P(y(n))}{\partial y(n)} r^{*}(n)
\end{aligned}
$$

The factor $1 / 2$ is used merely for convenience. The above algorithm might experience gradient noise amplification whenever $P(y(n))$ is large. Hence we normalized the correction term above by dividing with $a+P(y(n))$ with the choice of $a=1$. This idea comes from the well-known "normalized LMS algorithm," which can be viewed as the minimum-norm solution. The positive constant "a" is used to remove numerical difficulties that arise when the denominator is close to zero. Thus the equation (15c) becomes;

$$
w(n+1)=w(n)-\mu \frac{\partial P(y(n)) / \partial y(n)}{1+P(y(n))} r^{*}(n)
$$

With the expressions $[(y(n)+1)(y(n)-1)]^{2}$ and $[y(n)(y(n)+1)(y(n)-1)]^{2}$ for $P(y(n))$ respectively, we arrive at:

$$
\begin{gathered}
\psi_{3}(y(n))=\frac{4 y(n)\left[1-y^{2}(n)\right]}{1+\left[y^{2}(n)-1\right]^{2}} \\
\psi_{4}(y(n))=\frac{4 y(n)\left[1-y^{2}(n)\left[3 y^{2}(n)-1\right]\right.}{1+y^{2}(n)\left[y^{2}(n)-1\right]^{2}}
\end{gathered}
$$

Further, as the step size $\mu$ controls the rate of convergence, with a large value giving fast convergence and a smaller value providing better steady state performance we introduce a variable step size, $\mu$, as done in the case of traditional least mean square (LMS) algorithm [11]. We suggest $\psi_{5}(y(n))$ (proposed error function) as:

$$
\psi_{5}(y(n))=\mu(n) \psi_{4}(y(n))
$$




$$
\mu(n)=\lambda \mu(n-1)+\beta \psi_{4}(y(n))
$$

with $0<\lambda<1, \quad \beta>0$ and

$$
\mu(n)= \begin{cases}\mu_{\max }, & \text { if } \quad \mu(n)>\mu_{\max } \\ \mu_{\min }, & \text { if } \quad \mu(n)<\mu_{\min } \\ \mu(n), & \text { otherwise }\end{cases}
$$

where a condition of $0<\mu_{\min }<\mu_{\max }$ must be satisfied. The initial value of variable step size $\mu(0)$ is chosen according to the upper bound constant $\mu_{\max }$.

In equation (20), $\lambda$ and $\beta$ are two fixed parameters which control the variation of $\mu$ within two limits $\mu_{\text {min }}$ and $\mu_{\max }$. The updated equation in (8) with the step-size and error function as in (19) and (20) respectively is thus a variable step-size algorithm. The effectiveness of the three error functions (algorithms) with the usual CMA algorithm is shown through simulation in the next section.

\section{SimUlation CONSIDERATIONS}

To demonstrate the effectiveness of the error functions proposed which affect the speed of convergence of the equalizer, we assume that the transmitted signal is a zero mean distributed random sequence and is modulated using binary phase shift keying (BPSK) in which case the dispersion constant $\mathrm{R}$ is taken unity.

Also for the simulation the following parameters were used:

(i) a two-tap filter $w=\left[w_{0} w_{1}\right]^{T}$,

(ii) a constant step size $\mu=0.001$ and $\mu_{\min }=0.00001$ and $\mu_{\max }=0.1$ for variable step size algorithm,

(iii) $\lambda=0.97$, and $\beta=0.0005$, and equalizer initialization coefficients of 2 and 0.5 .

The additive noise after the channel is neglected, owing to the fact that in most digital communications the dominant type of distortion for which the equalizers are employed is the time varying multipath fading phenomenon, intersymbol interference (ISI).

Also, the BPSK modulation scheme used in this work is relatively immune to the additive noise levels present in communication channels.

\section{RESULTS}

Figures 2 to 4 show the plot of squared error against the iteration number for the usual Constant Modulus Algorithm (CMA) and the three different algorithms examined in this study.

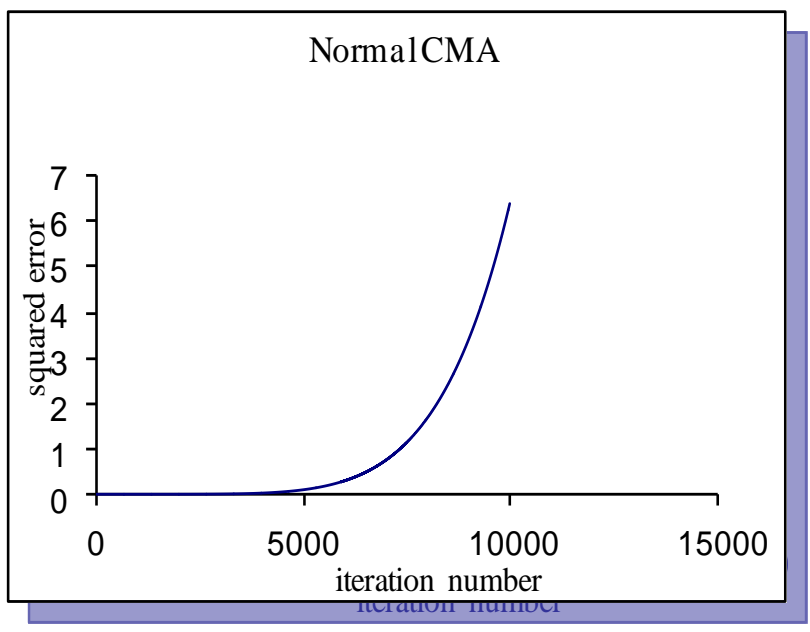

Figure 2: Squared error versus iteration for normal CMA

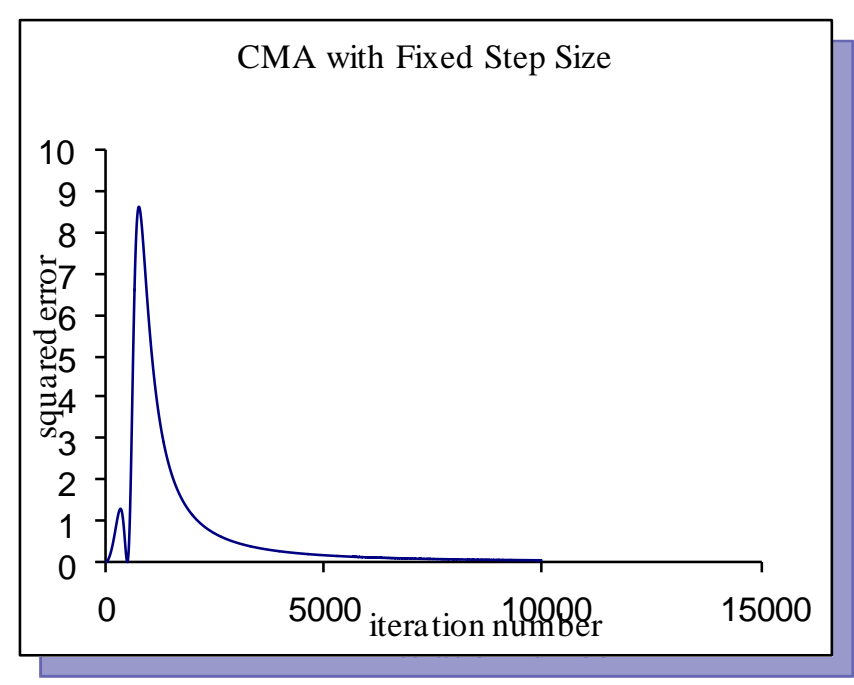

\section{Figure 3: Squared error versus iteration for CMA algorithm with step size 0.001 .}

The effects that the blind adaptive equalizer has on the signal quality are shown in Figures 2, 3, and 4. The algorithms aim at altering the filter impulse response so as to reduce and ultimately minimize the cost function. This is clearly seen in these Figures where the curves descend with time and then level out at their minimum. The time constant, or, more generally the convergence time of the algorithm, is indicated by the rate at which the cost function is reduced. 


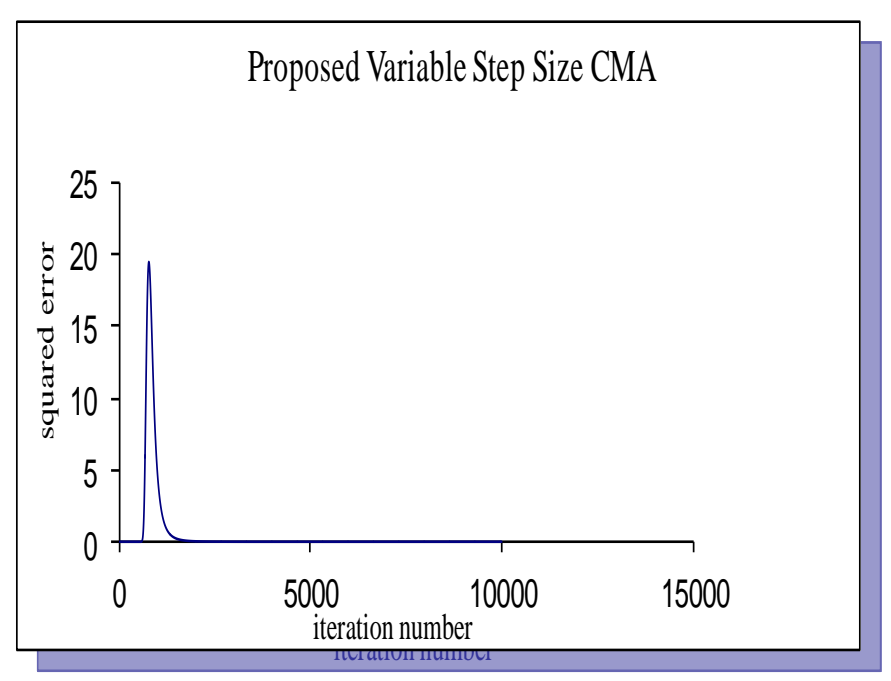

Figure 4: Squared error versus iteration for the proposed adaptive blind equalization algorithm with variable step size based on CMA criterion.

The Constant Modulus Algorithm (CMA) no doubts, does reduce the cost function, however, as observed in Figure 2, the normal CMA tends to diverge even after $1 \mathrm{msec}\left(10^{4}\right.$ iterations) of the time when the adaptation begins. This shows that it converges at some local minimum after a long time, comparatively. The algorithms with fixed step size shown in Figure 3 tend to continue improving the equalizer's performance even beyond $0.5 \mathrm{msec}$ point. The effect of the proposed algorithm with variable step size is quite obvious as can be seen in Figure 4.

The performance of this algorithm improves dramatically over the first few milliseconds and then flattens out. This variable step size algorithm appears to outperform all the other algorithms in this work because it ultimately minimizes the cost function to it minimum in a very short time, restoring the constant modulus of the transmitted signal, thus recovering the original signal. Note that the time it takes an algorithm to converge is significant: it is the measure of its ability to track the changing impulse response of the propagation channel.

From these results, it has been demonstrated that the equalizer, which employs this algorithm, does a good job of computing the new coefficient every $0.5 \mathrm{msec}$, which is good for fast varying channels.

\section{CONCLUSIONS}

The quality of service that a blind equalizer is able to provide is marked by its convergence speed; that is, the number of received samples that it needs to provide good enough estimates of the channel characteristics. This work has examined different error functions that might be incorporated into the Constant Modulus Algorithm (CMA) for fast convergence. The proposed variable step size algorithm outperforms the other two models in terms of convergence. The proposed algorithm can suitably be employed in blind equalization for rapidly changing channels as well as for high data rate applications. Based on our investigations blind method is a promising approach towards high data rate transmission and warrants further research in future communication technologies.

\section{REFERENCES}

[1] Kolawole, M. O. (2002). "Satellite Communication Engineering," New York: Marcel Dekker

[2] Ai, B., Yang, Z., Pan, C., Ge, J., Wang, Y. and Lu, Z. (2006). "On the Synchronization Techniques for Wireless OFDM Systems," IEEE Transactions on Broadcasting, vol. 52, no. 2, pp 236-244.

[3] Proakis, J. G. (2001). Digital Communication, Mc Graw-Hill Companies, Inc., International Edition.

[4] Adinoyi, A. Al-Semari, S. and Zerquine, A. (1999). "Decision Feedback Equalization of Coded I-Q QPSK in Mobile Radio Environments," Electron, Lett., vol. 35, pp. 13-14.

[5] Samueli, H., Daneshrad B., Joshi R., Wong B. and Nicholas H. (1991). "A 64-tap CMOS echo canceller/decision feedback equalizer for 2B1Q HDSL transceivers," IEEE Journal Selected Areas in Communication, vol.9, pp. 839-847.

[6] Iorkyase, E.T. (2010). A High Performance Blind Adaptive Channel Equalization Technique for Combating Intersymbol Interference in Digital Communication, MEng Thesis, Department of Electrical and Electronics Engineering, Federal University of Technology, Akure, Nigeria.

[7] Iorkyase, E.T. and Kolawole, M. O. (2010). "Comparative Study of Fixed and Variable Step Size Blind Equalization Algorithms in Communication Channels," International Journal of Electronics and Communication Engineering, vol. 3, no. 3, pp. 125-130

[8] Kazemi, S., Hassani H. R., Dadashzadeh G., and Geran F. (2008). "Performance Improvement in Amplitude Synthesis of Unequally Spaced array Using Least Mean Square Method," Progress in Electromagnetic Research B, vol. 1, pp. 135-145.

[9] Kundu, A. and Chakrabarty, A. (2008). "Fractionally Spaced Constant Modulus Algorithm for Wireless Channel Equalization" Progress in Electromagnetic Research B, vol. 4, pp. 237- 248.

[10] Nelatury, S. R. and Rao, S. S. (2002). "Increasing the Speed of Convergence of the Constant Modulus Algorithm for Blind Channel Equalization" IEEE Transaction on Communication, vol. 50. No. 6.

[11] Kwong, R. H. and Johnston, E. W. (1992). "A Variable Step Size LMS Algorithm,” IEEE Trans. Signal Processing, vol. 40, pp. 1633-1642. 